Research Article

\title{
Weight Control of Sports Training Chaos Predicting Model
}

\author{
Yanping Tang* and Guanghui Li
}

Institute of Physical Education, Langfang Teachers College, Langfang, 065000, Hebei, China.

Received 30 September 2014; Revised 20 October 2014; Accepted 15 November 2014

\begin{abstract}
As the sports training predicting model based on chaos local predicting method still has the low predicting accuracy and the slow function speed problems, this paper proposes a sports training chaos predicting model based on weight control ant colony algorithm. It firstly uses the comprehensive weight factor to perform the weight control to the initial information of the new join node in the ant colony algorithm, and then performs the optimizing iteration to the pheromone of weight control optimization, and updates the pheromone of ant node according to its contribution. Then the paper performs the optimization selection to the pheromone persistence parameters, the normalized factors and the relative importance factors to reduce the computing time and optimal error rate of the ant colony algorithm. Finally, adopt the improved ant colony algorithm to optimize the sports training chaos predicting model. The experiments show that the proposed sports training chaos predicting model based on weight control ant colony algorithm has a higher accuracy than the standard ant colony algorithm and the sports training chaos local predicting model.
\end{abstract}

Keywords: Sports training prediction, chaos local predicting model, improved ant colony algorithm, weight control, predicting accuracy, parameter optimization.

\section{Introduction}

It has become the main trend that the contemporary high technological theories and techniques deeply and competitively penetrate and engage in the field of sports training [1]. Scientific training plans and arrangements are based on the scientific test the analysis of the sports training, such as technical testing and analysis on sports, the processing and management on plenty of sport training information, and on this basis to summarize scientific training methods, which is an important part to achieve the fully control on the training process [2]. Advanced Science and technology can make an athlete's cultivation process more systematic, scientific and economic, which can fully excavate the athlete's potentials in all aspects and the best match them, making a breakthrough on the sports performance [3].

There are a few articles about sports predicting at home and abroad, and the range is relatively small. In the prediction research of the gold medal rankings at the Olympics, Song Shouxun adopts the comprehensive evaluation predicting method to the sport event of the Olympic Games in the big game take place in the cycle of the Olympics. On the basis of a comprehensive evaluation

* E-mail address: typli2004@163.com ISSN: 1791-2377 (C) 2015 Kavala Institute of Technology. All rights reserved. on the comprehensive and timely mastering all countries in the Olympic Games strength level and development, perform the medium and the recent prediction on the Olympic team pattern, gold medals, group strength rank, etc [4]. In the strength analysis and medal gold prediction of 2000 Sydney Olympic Game, Jin Haiquan adopts the sports expert method to perform the strength analysis of each country which are strong at diving, and predicts the situation that how many golden metals can China gets [5]. In the sports management and efficiency of prediction research, Wang Yue analyzes and process the date of economic benefits related to the games and activities through the analysis of some sports undertaking, summarizing some regular results, and adopts the analogy method to predict other sports events management and benefits [6]. In the application study of option event regression analysis in track, Liu Weimin use the two grades of the athletes body as the independent variable and dependent variable, establishing a regression equation to predict the results of the competition [7]. In the study of women's race walking athletes selecting and performance prediction, $\mathrm{Mu}$ Xiangdong uses stepwise regression method to find the key indexes which have the most closely relationship with the performance of the sport so as to obatin the best regression equation and discriminant function, which will provide a scientific basis for the women's race walking athletes selecting [8]. In the study of GM $(1,1)$ model and its prediction of the women's $400 \mathrm{~m}$ hurdles world and the 
national record, Tong Liping predicts the women's $400 \mathrm{~m}$ hurdles world record through constructing the model of GM $(1,1)$ [9]. In the study of markov chain and its predicting model of woman athletics achievement in our country, He Qiuhua predicts the achievements of China's women's track and field sports through establishing the prediction model of markov chain [10]. In the design research of the long jump athletes' index predicting model in colleges, Zhou Ming performs a qualitative analysis to the factors of long jump, selects 22 indicators, uses the fuzzy relationship matrix, stepwise multiple linear regression method to perform the index selection, retains the nine items to establish return jump performance prediction model, and performs the prediction study for the long jump performance of student $\mathrm{Li}$ [11]. In the study of the two kinds of predicting methods of the sports, Chen Zhiqiang et al. uses factor analysis and grey system theory to improve the regression predicting model aiming at the defects of the current sports statistics the multivariate regression predicting model, and improves the accuracy and feasibility of the sports scientific prediction [12]. Liu Jiajin et al. put forward the theoretical basis for using GM (1, 1) model in the sports prediction, and combined with the best grades of the men's hammer throw, from two aspects of theory and practice to GM $(1,1)$ model of prediction in the sports, performed detailed research, and discussed some restrictive factors exist in the application [13].

In view that the accuracy of the general chaos predicting model is not high, this article designs a kind sports training chaos predicting model based on weight control ant colony algorithm, adopting the weight control strategy to improve the initialization and update of the pheromone in ant colony algorithm and then optimizing the selection of its parameters, as well as improving the accuracy of the sports training chaos predicting model.

\section{Defects of General Chaos Prediction Model}

Through the training function monitoring to detect and assess the status of athlete bearing the training load is an important means of diagnosis and monitoring the athletic ability, and it is also an important part in today's scientific training. This paper uses the chaos forecasting method to construct the prediction model in sports training. The general chaos predicting model can be divided into chaos global domain predicting method and the chaos local predicting method:

(1) The chaos global predicting method.

For time series $\{X(t)\}, t=1,2, \ldots, N$, the phase space state vector can be obtained after reconstruction:

$$
X(t)=\{x(t), x(t-\tau), \ldots, x[t-(m-1) \tau]\}
$$

According to the given data structure mapping function $F$, make the future state $X(t+1)$ and current

state $X(t)$ satisfy:

$$
X(t+1)=F(X(t))
$$

where, the constructing criterion of the mapping function $F$ makes the following formula obtain the minimum value.

$$
\sum_{t=0}^{N}\{X(t+\eta)-F(X(t))\}^{2}
$$

In the process of phase space reconstruction, if the dimension is low, the high order polynomial, generally, can be directly used to perform the global matching; And for high dimensional phase space, its computational complexity are multiplied, which generally uses the typical regression analysis model to minimize the amount of calculation:

$x(t+1)=\sum_{i=1}^{d} a_{i} x(t+1-i)+k \varepsilon_{t}$

where, $\varepsilon_{t}$ is Gaussian random variables, which obey the standard normal distribution $N(0,1) ; k$ is the constant factor which adjusts the random intensity, which generally takes $k=\sqrt{E_{d} /(N-d)} ; a_{i}$ can be obtained by time series itself. In order to ensure the influence of random input on the system as small as possible, $a_{i}$ needs to make the sum of the system error square minimum;

$$
E_{d}=\sum_{t=d}^{N}\left[x(t)-\sum_{i=1}^{d} a_{i} x(t-i)\right]^{2}
$$

Perform the partial derivatives to $a_{i}$ and make the result zero, so:

$$
\sum_{i=1}^{d} a_{i}\left[\sum_{t=d}^{N}[x(t-i) x(t-j)]\right]=\sum_{t=d}^{N}[x(t) x(t-j)]
$$

After the deformation:

$\sum_{i=1}^{d} a_{i} C(i-j)=C(j)$

where, $C(i-j)$ and $C(j)$ are the autocorrelation functions, so the coefficient $a_{i}$ is obtained.

It can be seen from the above analysis that when the embedding dimension is higher or the phase trajectory is complex, the calculation of global domain method is more complicated, and accuracy will also fell sharply with the increase of the embedding dimension, so the global domain method is suitable for the situation that the prediction function is simpler and the interference is less.

(2) The chaos local predicting method.

Assume the chaos time series $\{x(t)\}$, and perform the phase space reconstruction to its one-dimensional time series by choosing the embedding dimension $m$ and delay time $\tau$, the state of the phase space $\{X(t)\}$ can be obtained at time $t$, including:

$$
X(t)=[x(t), x(t-\tau), \ldots, x(t-(m-1) \tau)]^{T}
$$

$M=N-(m-1) \tau$

The steps of the local predicting method can be summarized as follows: firstly, find the $K$ state points $\{X(t)\}$ which are the closest to the state trajectory $X(t)$. Then perform the judgment, if the system is determined, so when 
$X(t)$ gets close to $X\left(t_{i}\right)$ and $X(t+1)$ gets close to $X\left(t_{i}+1\right)$, the average of the $X\left(t_{i}+1\right)$ is the predicting value of the trajectory point in the next step:

$$
X(t+1)=\frac{1}{N} \sum_{i=1}^{N} X\left(t_{i}+1\right)
$$

After reconstructing the phase space, when search for the neighbourhood, consider distance factor between the adjacent point in the neighbourhood and the center point, which can improve the predicting performance in theory. Adopt the improved method - the weighted local predicting method, and its expression is as follows:

$$
\hat{X}(t+1)=\frac{\sum_{i=1}^{N} X\left(t_{i}\right) e^{-l\left(d_{i}+d_{m}\right)}}{\sum_{i=1}^{N} e^{-l\left(d_{i}+d_{m}\right)}}
$$

where, $\hat{X}(t+1)$ presents the point which needs to be predicted; $X\left(t_{i}\right)$ present the points around the center point $X(t)$ in the neighbourhood; $N$ presents number of the points in the neighbourhood; $d_{i}$ presents the distance between the adjacent point and its center point in phase space, $d_{m}=\operatorname{mid}\left(d_{i}\right)$ presents the minimum distance between adjacent points; $l$ is a parameter, generally taking $l \geq 1$.

Comparing to the global domain predicting method, although the accuracy of the weighted local prediction method has a great improvement, its anti-interference is still very poor, so this paper adopts the improved ant colony algorithm to optimize the chaos predicting model.

\section{The Chaos Predicting Model Based on Improved Ant Colony Optimization}

\subsection{The Defects of Ant Colony Algorithm in the Predicting Model}

In the standard ant colony algorithm, set the $\tau_{i j}$ as the trajectory intensity of the path $(i, j), \Delta \tau_{i j}^{k}$ as the amount of the unit trajectory pheromone leaved by the ant $k$ in the path $(i, j), \quad p_{i j}^{k}$ as the transfer probability of ant $k$, $a(a \geq 0)$ as the relative importance of the trajectory, $\beta(\beta \geq 0)$ as the relative importance of the path visibility and $\rho(0 \leq \rho<0)$ as the residual degree of the pheromone. The determination of these parameters is the decisive factor of the implementation of the algorithm and the algorithm efficiency.

The expressions of $\tau_{i j}, \Delta \tau_{i j}^{k}$ and $p_{i j}^{k}$ can be different, which are determined by the specific problem. In general, the transition probability of ant $k$ is:

$$
p_{i j}^{k}=\left\{\begin{array}{cc}
\frac{\left[\tau_{i j}\right]^{\alpha}\left[\eta_{i j}\right]^{\beta}}{\sum_{u \in \text { allowed }_{k}}\left[\tau_{i j}\right]^{\alpha}\left[\eta_{i u}\right]^{\beta}} & \text { if } j \in \text { allowed }_{k} \\
0 & \text { otherwise }
\end{array}\right.
$$

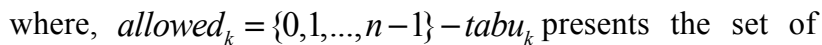
the choice by the $k$-th ant in the next step. $a$ and $\beta$ respectively present the information accumulated by the ant in the moving process and the influence of the heuristic factor on the ant's decision-making.

The updating equation of the trajectory intensity is:

$\tau_{i j}^{\text {new }}=\rho \cdot \tau_{i j}^{\text {old }}+\Delta \tau_{i j}$

shere, $\Delta \tau_{i j}=\sum_{k=1}^{m} \Delta \tau_{i j}^{k}$, the amount of unit trajectory pheromone of the ant $k$ is $\Delta \tau_{i j}^{k}$.

However, the standard ant colony algorithm has many defects, such as the long computing time and easy to emerge stagnation etc., so this paper improves the ant colony algorithm before using it to optimize the chaos predicting model.

\subsection{Ant Pheromone Optimization Based on Weight Control}

In the improved ant colony algorithm, the size of the pheromone on the optional path determines the probability of the ant routing. The continual change of the pheromone play an important role for the ants to select the optimal path, so this paper use weight control to initialize and update the pheromone:

(1) When there is a new athlete $i$ joins the predicting model, the athlete node $i$ in the model needs to inform its training parameters to all neighbor nodes $j$, and the neighbor nodes add a new compared directory, set the hop number section 1. The initial pheromone on the $v_{i j}$ is related to comprehensive weighting factors $e_{i}$ and $e_{j}$ of the node $i$ and node $j$. Initial pheromone of the on the $v_{i j}$ is as follows:

$$
\tau_{i j}(0)=e_{i} \cdot e_{j}
$$

In this article, after the initialization of the pheromones on each path, perform the normalization to the pheromones, which is mainly to adjust weights of the increment between the pheromone on the path after the last iteration and the pheromone on the path of the current iteration.

(2) After each iteration, the algorithm needs to update the concentration of pheromone on the path according to nodes passed by the ants, the updating way of the pheromone on $v_{i j}$ is as the following:

$$
\tau_{i j}(t+1)=\rho \cdot \tau_{i j}(t)+\Delta \tau_{i j}(t, t+1)
$$

In formula (15), $\Delta \tau_{i j}(t, t+1)$ presents the change of the pheromones on the $v_{i j}$ in the searching process.

$\Delta \tau_{i j}(t, t+1)=\sum_{k=1}^{m} \Delta \tau_{i j}^{k}(t, t+1)$

In formula (16), $m$ presents the number of the ant, presents the contribution of each ant to the pheromone on the path $v_{i j}$ in that iteration process. 
$\Delta \tau_{i j}^{k}(t, t+1)= \begin{cases}\frac{e_{i} \cdot e_{j} \cdot \varepsilon_{i j} \cdot H}{h^{\prime} s_{i j}} & , j \in \text { next }_{k} \\ 0 & , \text { otherwise }\end{cases}$

where, $H$ presents positive constant, which is related to the hop number hops $_{i j}$ between the nodes in the predicting model. When there is no information of the wanted comparing parameters in the node $i, \varepsilon_{i j}$ takes 0.5 .

(3) For the parameters of the athlete nodes in the model comparing with each item in the catalogue, they will be regularly updated, the updating formula is as follows:

$\tau(t+1)=\rho \cdot \tau(t)$

Therefore, when a sport parameter was not visited for a long time, the concentration of the corresponding pheromone will be close to 0 , which means the athlete parameters provided by the athlete node in this model is less used, or that node is inaccessible.

(4) When the ant find the wanted athlete parameter on the last node in a routing, the pheromone concentration between the link of the nodes which are connected with that node multipliesn $\tau_{e n}$, which will increase the pheromone concentration leading to the effective node on the path;

(5) When the ant does not find the wanted athlete parameter on the last node in a routing, the pheromone concentration between the link of the nodes which are connected with that node divides $\tau_{p u}$, which will decrease the pheromone concentration leading to the effective node on the path;

(6) If the ant does not find the parameter information on the target athlete node according to the athlete parameter of the athlete node on the path in the predicting model, then according to the formula(13), updates the pheromone on that path which is passed by the ant;

(7) Same as the pheromone initialization, perform the normalization to the information increment;

(8) Add the pheromone increment to the original pheromone, also performs the normalization.

In the improved ant colony algorithm, the routing mechanism of the ant are: first of all, choose the next optional hop node set next $k_{k}$ according to $t a b u_{k}$ and $A T_{k}$, and then according to the transmitting probability:

$$
p_{i j}^{k}(t)=\frac{\tau_{i j}^{\alpha}(t) \cdot \eta_{i j}^{\beta}(t)}{\sum_{u \in n_{n} t_{k}} \tau_{i u}^{\alpha}(t) \cdot \eta_{i u}^{\beta}(t)}
$$

Select the next hop node $j$. If the next $t_{k}$ is an empty set, presents the next optional hop node is empty, so the ant "die" in that node and no longer transmits.

In order to control the number of ants in the network, each ant carries a survival time field $(T)$, and when the ant passing a node, the field value will reduce 1 . When $T=0$, the ant will no longer transmit. Setting the appropriate $T$ can set the scope of the routing. When $T$ is small, the searching scope is small, whereas when $T$ is bigger, the searching scope is larger.

\subsection{Optimization of Parameter Selection}

After performing the weight control to the standard ant colony algorithm, executes the selecting optimization to the parameter.

(1) The selection of the pheromone persistence parameter $\rho$.

The selection of $\rho$ determines the importance of the prior knowledge of the ants in routing: when $\rho$ is small, the concentration of the pheromone on the path, which is not selected or less selected, will decrease rapidly after the updating of the pheromone. In the next routing, the following ants tend to walk on the selected path, which will lead to a drop in the ant searching scope and make the algorithm trapped in local optimal solution early, but the convergence speed of the algorithm will be fast. Otherwise, when $\rho$ is big, the concentration of the pheromone on the path, which is not selected or less selected, will decrease slowly, and the following ants tend to walk on the unselected or less selected path, which will increase the searching scope. So, the algorithm is not easy to trap in local optimal solution, but due to the increase in the number of paths optional, the convergence time/number of iterations will increase.

At the beginning of the improved ant colony algorithm, as the global parameters of the predicting model are unknown, it is need to consider that the optimal path need to be selected from a lot of alternative paths. $\rho$ should be large to expand the range of ants routing and find the different paths which can reach the target athlete node. After a certain number of iterations, the difference of pheromone is big on the paths, so the convergence speed of the algorithm can be accelerated, and $\rho$ should be set a small value. In this article, $\rho$ is set to be the exponential function of the current iteration number $n_{i t}$, the expression of $\rho$ is as shown in formula (20). Therefore, the $\rho$ is big at the beginning of the improved ant colony algorithm, and the ant's searching scope is large. With the increase of the number of iteration, $\rho$ will decrease, which improves the convergence speed of the algorithm.

$\rho=\rho_{0} \cdot e^{-n_{i t} / N_{i t} \cdot 10}\left(0 \leq n_{i t} \leq N_{i t}\right)$

where $0<\rho_{0}<1, N_{i t}$ is the total number of the iteration. $\rho_{0}$ is the initial value of $\rho$, which takes a big value.

(2) The selection of normalized factor.

When $H$ is big, the pheromone increases rapidly, and the improved ant colony algorithm may converge to the local optimal solution. When $H$ is small, the positive feedback information in the process of each pathfinding is little, and the algorithm is not easy to converge, so the pheromone is little incentive. This article set $H$ according to the matrix of $h_{o p s_{i j}}$ of the node in the sports training predicting model.

(3) The selection of the relative importance factor $a$ of the pheromone $\tau_{i j}$ and the relative importance factor $\beta$ of the visibility factor $\eta_{i j}$

The routing probability of the next moment is related to the pheromone concentration $\tau_{i j}$ of each optional path, the visibility $\eta_{i j}$ of the path and the value of $\alpha$ and $\beta$. When $\alpha$ is big, the pheromone concentration $\tau_{i j}$ is in a dominant position on the ant routing probability, and the ant tends to choose a path with more pheromone and the routing mechanism focus on the global optimization. When $\beta$ is big, the effect of the distance between the adjacent nodes on the ants routing increases, the routing mechanism focus on the local optimization. 
Based on the above analysis, in the early part of the improved ant colony algorithm, the ant finds few paths and mainly relies on adjacency relations to choose the path, so $\beta$ should be set a large value. In order to avoid the improved ant colony algorithm trapped in local optimal solution, the influence of the pheromone $\tau_{i j}$ on the ant's routing probability should be increased, and the value of $\alpha / \beta$ should be increased compared with the early stage of the algorithm. So, the value of $\alpha / \beta$ will be increased appropriately by increasing $\alpha$ or reducing $\beta$.

In order to simplify the selection and settings of the parameters, $\alpha$ will be set to a fixed value in this article, the initialized value of $\beta$ is bigger than $\alpha$. The value of $\beta$ decreases with the increasing of iterations, in this way, in the early stage of the improved ant colony algorithm, the value of $\alpha / \beta$ is small;

Late in the improved ant colony algorithm, the value of $\alpha / \beta$ is bigger, and function relation between $\beta$ and $n_{i t}$ is as shown in formula (21).

$$
\beta=\beta_{0}\left(1-n_{i t} / N_{i t}\right)
$$

In formula (21), $n_{i t}$ presents the current iteration number, $N_{i t}$ presents the total number of iteration in the improved ant colony algorithm, and set $\alpha=0.5 \beta_{0}$ in this article.

\subsection{The Chaos Predicting Model Based on Improved Ant Colony}

Adopt the improved ant colony algorithm to optimize the chaos predicting model, and update the pheromone of the single ant after the searching optimization as follows:

$c_{i}=\varepsilon_{i} p_{1 i}=\left(1-\varepsilon_{i}\right) p_{2 i}$

where, $p_{i j}$ is the optimization probability of the ant initial value $p, \varepsilon_{i}$ is a random number between $[0,1]$.

$$
c_{i}^{\prime}=c_{i}+\varepsilon_{i} \lambda_{i} \exp \left[-\gamma(t-1)^{\beta} / \omega\right]
$$

$t$ presents the current number of iteration, $\lambda_{i}$ presents the largest step of variables $x_{i}$ in $i$-th interval, $\beta$ and $\omega$ are the parameters controlling the attenuation rate of the nonlinear steps. $\varepsilon_{i}$ and $\gamma$ are the random numbers between $[0,1]$.

$$
\tau(c)=1 / n \sum_{i=1}^{n}\left[\varepsilon_{1} \tau_{p 1}+\left(1-\varepsilon_{i}\right) \tau_{p 2}\right]
$$

Then, use the chaos sequence to produce several test points in order to traverse the entire searching interval, and the initial colony determines the initial pheromone of each region according to the optimal value of each region. The algorithm can also make use of the chaos system to produce a large number of test points as the working ant colony. In order to find out the new optimal solution with the chaos algorithm, the working ant colony randomly selects the areas around the variables which are corresponding to the optimal ants to search based on the amount of the pheromone in different areas. So, the global optimal solution will be found within a specified number of iterations.

So, the basic steps of the chaos predicting model based on improved ant colony algorithm are as follows:
(1) Initializing set the iteration number $N$ and the number $m$ of the ants.

(2) Put $m$ ants in the searching range, and perform path searching for each variable.

(3) Perform the global searching process, and analyze the ant which finishes the global path searching, and update the path and the pheromone.

(4) Select the global optimal ant.

(5) Enter the local searching process of the ant colony optimization, make the quantity of the ant decrease with the increasing of the iteration number and then contract to global searching range, at the same time update the pheromone.

(6) Enable the chaos optimization algorithm to perform the chaotic search near the current optimal solution, namely the global optimal ant, and if the current best solution is found, the original optimal solution is replaced by it.

(7) If $m$ ants do not finish the analysis, skip to step (3).

(8) Update the pheromone on all the routes passing by each ant:

$$
\tau_{k} \leftarrow \rho \cdot \tau_{k}+\Delta \tau_{k}, \Delta \tau_{k} \leftarrow 0
$$

(9) If the iteration is not finished, skip to step (2).

(10) Finish all the iterations, and output the predicting value.

\section{Performance Simulation of the Algorithm}

In order to verify the effectiveness of the improved algorithm proposed in this paper, perform simulation experiments on it. First of all, respectively use the improved ant colony algorithm and the basic ant colony algorithm to perform the optimization to the following four standard test functions. In this experiment, choose 50 ants, the maximum number of iterations is set to 50 times, the cycle number $N$ is set to 50 times.

(1) Function $f_{1}$

$$
f_{1}(x)=x_{1}^{2}+2 x_{2}^{2}-0.3 \cos \left(3 \pi x_{1}\right)-0.4 \cos \left(4 \pi x_{2}\right)+0.7
$$

(2) Function $f_{2}$

$$
f_{2}(x)=\sum_{i=1}^{n-1} 100\left(x_{i+1}-x_{i}^{2}\right)^{2}+\left(1-x_{i}\right)^{2}
$$

(3) Function $f_{3}$

$$
f_{3}(x)=10 n+\sum_{i=1}^{n}\left(x_{1}^{2}-10 \cos \left(2 \pi x_{1}\right)\right)
$$

(4) Function $f_{4}$

$$
f_{4}(x)=1+\sum_{i=1}^{n}\left(\frac{x_{1}^{2}}{4000}\right)-\prod_{i=1}^{n}\left(\cos \left(\frac{x_{i}}{\sqrt{i}}\right)\right)
$$

The above four functions are relatively typical testing functions, and the testing results are shown in Figs. 1-4. 


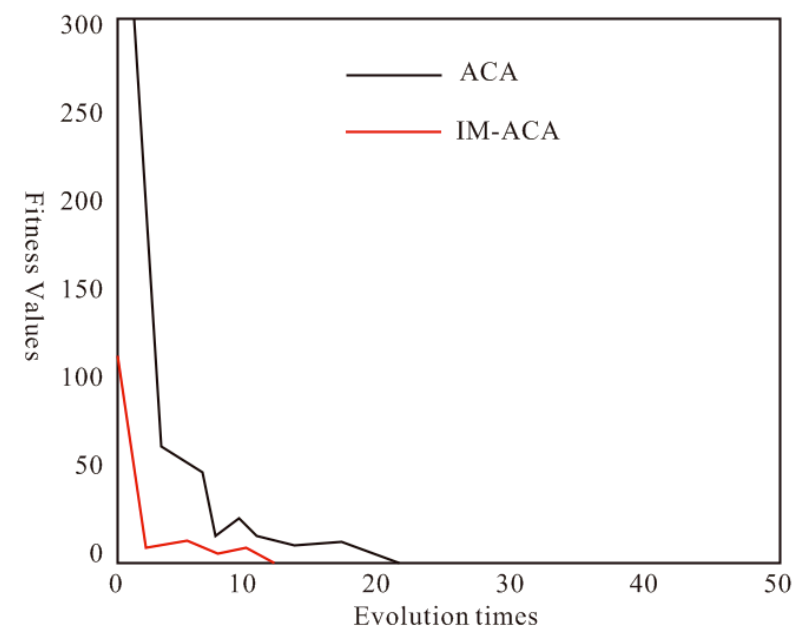

Fig. 1. Function $f_{l}$ optimization results.

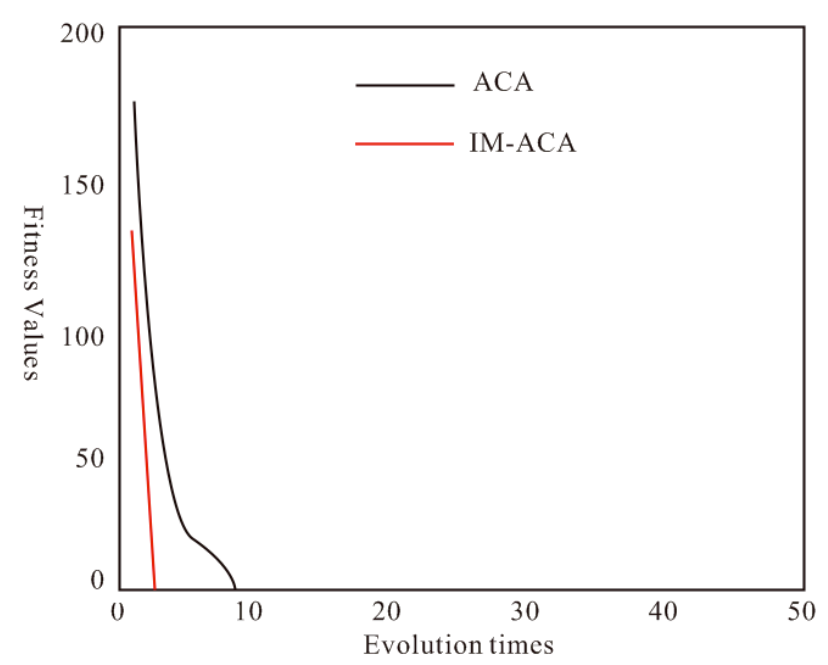

Fig. 2. Function $f_{2}$ optimization results.

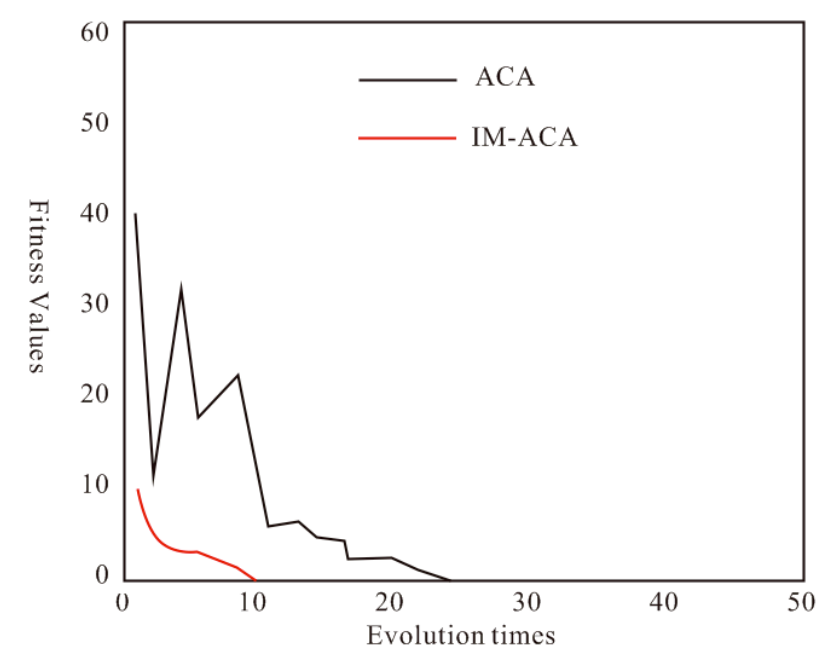

Fig. 3. Function $f_{3}$ optimization results.

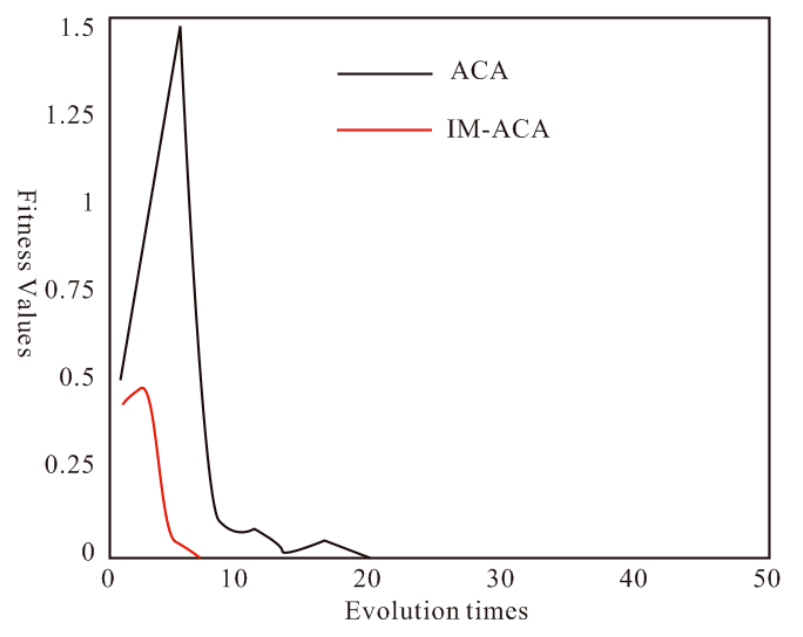

Fig. 4. Function $f_{4}$ optimization results.

It can be seen from the above simulation results that compared with the original ant colony algorithm, the improved ant colony algorithm proposed in this paper has a higher accuracy and a better convergence performance.

Then, apply the improved ant colony algorithm to sports training predicting model, compare it with the standard ant colony algorithm and the chaos local predicting model algorithm, and test the predicting error, the results are as follows:

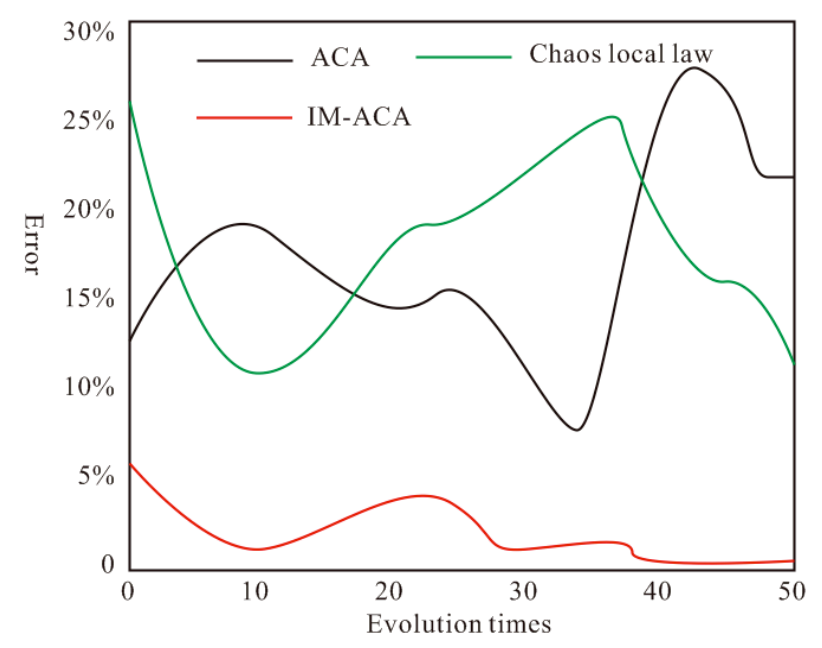

Fig. 5. Sports training prediction error contrast.

It can be seen from the simulation results that compared with ant colony algorithm and chaos local predicting model, the proposed sports training chaos predicting model based on weight control ant colony algorithm has a higher predicting accuracy.

\section{Conclusion}

In sports training, as the internal and external environment changes a lot and the pheromones are complex, the entire training is in a chaotic state, therefore, the sports 
training must be predicted to the determine the occurrence, development and trends of the uncertainty as far as possible and make reliable dynamic control to the sports training in advance. In this paper, the sports training chaos predicting model based on weight control ant colony algorithm is proposed, and the simulation results show that the model has a high predicting precision and a good adaptability.

\section{References}

1. Z. Jiabei, Recent projections of world basketball championship final grades, Journal of Wuhan Sports College, vol. 3, pp. 54-59 (2014).

2. X.U. Yijun, Application status of the fuzzy neural network in prediction of welding performance, Electric Welding Machine, vol. 44(6), pp. 46-50 (2014).

3. D. Lijun, All items of track and field sports development forecast, Sports and science, vol. 4, pp. 8-9 (2014).

4. Z. Airong, BP neural network prediction method of gas concentration based on the wavelet analysis, Coal mine machinery, vol. 35(6), pp. 258-260 (2014).

5. X. Xiangjun, Universal prediction and selection of athletes, The Chinese sports science and technology, vol. 12, pp. 25-26 (2013).

6. C. Guimei, Predictive Modeling of Blast Furnace Temperature by Using Distributed Neural Network Model, Journal of Iron and Steel Research, vol. 26(6), pp. 27-30 (2014).

7. C. Yunlong, The relation between triple jump distance, proportion and the performance and performance prediction. Journal of Wuhan Sports College, vol. 3, pp. 6-13 (2014).
8. F. Zhaofeng, RBF neural network multi-step predictive control for nonlinear systems, Control and Decision, vol. 29(7), pp. 1274-1278 (2014).

9. L. Jianguo, Fosbury style high jump performance forecast and control training standards, Chinese Sports Science and Technology, vol. 23, pp. 28-32 (2014).

10. H. Min, Research on multivariate chaotic time series prediction using mRSM model, Acta Automatica Sinica, vol. 40(5), pp. 822-829 (2014).

11. W. Yang, Transmission model of micro-blog network information based on gray prediction, Journal of Anhui Normal University (Natural Science Edition), vol. 37(2), pp. 129-133 (2014).

12. L. Yang, Based on the least square method in aerobics training intention and behavior prediction research, Modern sports technology, vol. 3(34), pp.4-5 (2013).

13. X. Wu, Review of sports prediction method, Journal of Shandong Sports Science and Technology, vol. 23(1), pp. 62-64 (2012). 